\title{
Launching extinguishing carriers into remote forests for active fire fighting
}

\author{
M. Boboulos ${ }^{1}$, M. Purvis ${ }^{2} \&$ D. Zafirov ${ }^{3}$ \\ ${ }^{1}$ Department of Mechanical Engineering, \\ Technical Education Institute of Larissa, Greece \\ ${ }^{2}$ Department of Mechanical and Design Engineering, \\ University of Portsmouth, UK \\ ${ }^{3}$ Transport and Aircraft Equipment Technologies, \\ Technical University of Sofia, Bulgaria
}

\begin{abstract}
Conceptual research considering the option to fight forest fires by designing a carrier-body containing extinguishing agents that can be shot from a distance into the fire zone is presented in the current paper. This active method can be applied in situations where conventional fire-fighting cannot be employed, to remotely deliver extinguishing mixtures from a $2.5 \mathrm{~km}$ distance, through controlling launching parameters as the starting angle and the shooting direction. The launching and flight of a projectile and a missile variant have been examined in this paper. Simulations for the variants in actual atmosphere have been produced, to obtain correlations between body parameters and its performance. Thus, aerodynamic causes and their effect in the body's performance were determined. A design evaluation has been prepared to determine optimum body parameters, stability and geometry for the most suitable carrier design.

Keywords: extinguishing agents, aerodynamic coefficients, optimal body shape, flight stability, trajectory dynamics, launching parameters.
\end{abstract}

\section{Introduction}

Fire and ecosystems have co-existed for millennia and they will continue to do so. There is, however, a continuous change in their balance with a tendency for a fire increase and a forest reduction, due to human's negligence or deliberate causing [1]. In recent decades, there has been a dramatic increase in both the fire 
number and the burnt areas in many countries [2]. The problem is converged in that, when fires occur in hard to reach areas fire-fighting transportation and equipment have limited use, since they cannot deliver their agents over $250 \mathrm{~m}$ in length or in a dissimilar pattern. Airplanes cannot be applied when strong winds are present or during the night.

Fire fighting was greatly developed in the previous years, redesigned aircrafts and vehicles, equipment and agents provided efficient assistance [3]. All these introductions improved the fire fighting in hard reached areas. A reputable need however, to remotely deliver extinguishing mixtures from a distance by controlling the launching can nonetheless be found. The solution comes in developing a carrier and a system capable of delivering agents, with a view for correlating body parameters and its performance. The research objectives therefore associated to: a) Provide clarification on the carrier's flight to the fire zone; b) Designing a suitable carrier body and system; c) Checking the targeting accuracy of the movement's model through firing simulations.

It has been supposed that quick action at fire initiation would provide support for immediate fire put out, using insignificant agent volumes. The investigation follows McCormick's flight dynamics work relating to aerodynamic concepts of a carrier's movement in actual atmosphere, describing a body's low drag and stable flight [4]. When actual environment deviation is recorded, the launching data will have to be recalculated before each individual shooting. Processing can be done with simulation and against launching statistics, from aerodynamic experiments [5] and trial launching [6].

\section{Physical modelling}

Body technical requirements were determined by the fire system as a whole. The carrier requirements were for: firing 500-2,500m; agent weight above $2 \mathrm{~kg}$; wind speed up to $120 \mathrm{~km} / \mathrm{h}$ and temperature $\geq 45^{\circ} \mathrm{C}$. Two carriers fit correspondingly to these requirements, the missile and the projectile [10].

\subsection{Technical flight requirements}

Body parameters influencing the system were geometric, the empty body weight, system's thrust, body's shape, and fuel weight. Travel distance, flight time, net volume, and fire agent weight were influential in a product's development [11].

\subsubsection{Functional and structural analysis}

Fire agent delivery concentrated into the arrangement shown in Figure 1. The carrier is fired to follow a specified trajectory and its body splits into two at a specified height to spray the extinguishing agent over a specified surface. This necessitated providing a squib and filler charge for both carriers. The squib sent out a signal that actuated the filler charge to spray the fire-extinguishing agent over the fire. The carrier body incorporated all elements and provided the required net volume for the agent. The body consists of two sections, which split when actuated by the squib to release the agent, as shown in Figure 2. Therefore, 
the connection between the body's two sections is a critical area having a belt shape, ensuring the splitting, and better agent spraying. Flight stabilisation for the missile carrier is provided by stabilisers [10]. Projectile gyroscopic stabilisation is accomplished by rotating in the longitudinal axis [6].

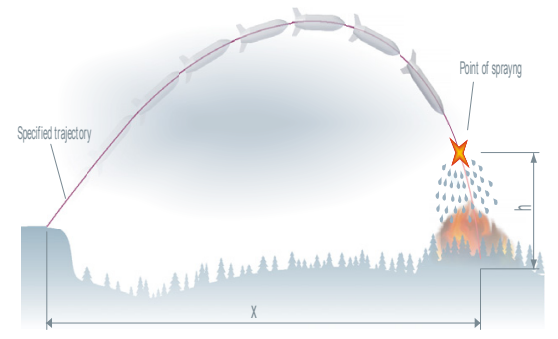

Figure 1: Missile's flight.

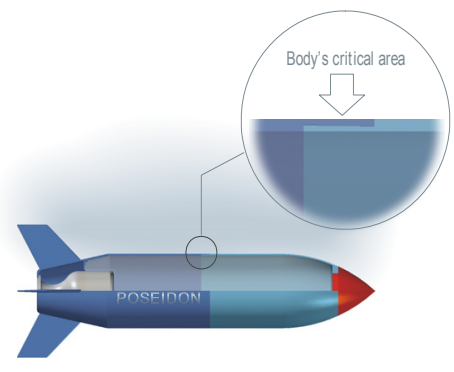

Figure 2: Connection point between body sections.

\subsubsection{Forces}

Aerodynamic forces acting on the projectile flight are influenced by air density. Therefore, an atmospheric model has been developed ensuring target hitting precision and reducing the carrier number for fire put out. Since the movement of a stabilised uncontrollable carrier takes place under small launching angles $(\alpha)$, the lift force $(\mathrm{L})$ is negligible and can therefore be disregarded. The drag force on the carrier depends on air density, the drag factor, the outer diameter size (calibre) and flight velocity [5]. Drag force reduction is achieved by selecting a suitable carrier shape, optimum elongation and a carrier featuring minimum difference between the velocities along the specified trajectory [6].

\subsection{Surrounding environment}

\subsubsection{Gravitational field}

A model of flat parallel gravitational field has a negligible effect on the calculation accuracy, due to the low value of the maximum travelled distance. A gravitational field model corresponding to the geometrical earth shape is usually adopted for distances $\leq 100 \mathrm{~km}[10]$. On the same grounds the variations in earth's acceleration have been disregarded when travelling in different geographical latitudes [12]. The model uses the earth's acceleration value, corresponding to the geographical coordinates of the launching mechanism [11]. From a desired hitting precision viewpoint this is acceptable, given the fact that the maximum relative trajectory height is never above $2,000 \mathrm{~m}$ [11].

\subsubsection{Atmosphere}

Anderson [11] proves that atmospheric parameters with significant effect on flight distance and carrier drift were the air density, wind speed and direction. Low air densities resulting from high temperatures, low atmospheric pressure or 
high altitude of the launching position, usually increase flight distances. It has been assumed that the longitudinal and transverse projectile movements were independent of each other and consequently, wind speed has been decomposed into longitudinal and transverse velocity components. Longitudinal tail wind increases the flight, while longitudinal contrary wind decreases flight distance. Cross wind causes the carrier's trajectory to shift in the wind direction [8].

Ground-level air temperature, at the launching position has indirect effect on the initial temperature of the projectile's firing filler charge and the missile's motor fuel [11]. Initial launching speed and thrust are strongly influenced by this temperature [8]. Increasing the temperature of the firing filler charge increases the initial velocity hence, increasing flight distance. Raising the motor's fuel temperature, increases the engine thrust. The engine's operation time is reduced, which in turn increases the thrust pulse and the flight distance. In the simulations the fuel temperature has been measured, and at the moment of firing, this temperature is assumed to be the equal of the ground-level air temperature [8].

\section{Operational design}

\subsection{Body parameters and characteristics interrelations}

Geometric parameters influence body characteristics as the largest diameter (calibre) has a strong effect on the applied drag. The drag force is directly proportional to the calibre size's second power [13].

$$
F_{\text {drag }}=C_{D}(\alpha) A \frac{\rho V^{2}}{2}
$$

As a result the smaller the calibre, the smaller the drag and hence, the smaller the initial projectile velocity required and the smaller the missile fuel quantity requirement for identical extinguishing agent. An optimum ratio exists between body's length and the calibre, resulting in the lowest expenditure involved in a single firing operation [2].

$$
\lambda \text { opt }=1 / \mathrm{d}
$$

The geometric relationships between $\mathrm{d}, \mathrm{V}, 1$ and $\lambda$ opt determine 1 for a given d, V and $\lambda$ opt. An additional missile parameter is the thrust impulse, characterizing maximum body flight distance. Generally the relationship $P=f(t)$ has a complex plot shape [10]. As an approximation it has been assumed that the thrust impulse is of rectangular shape as shown in Fig. 3. Hence thrust is expressed by a $\mathrm{P}_{\mathrm{av}}$ average value. A fundamental projectile parameter is its initial speed. The larger the projectile mass, the lower the initial speed to achieve the pre-set distance. A $200 \mathrm{~m} / \mathrm{sec}$ initial speed would ensure carrier travel between 9 and $97 \mathrm{~kg}$. 


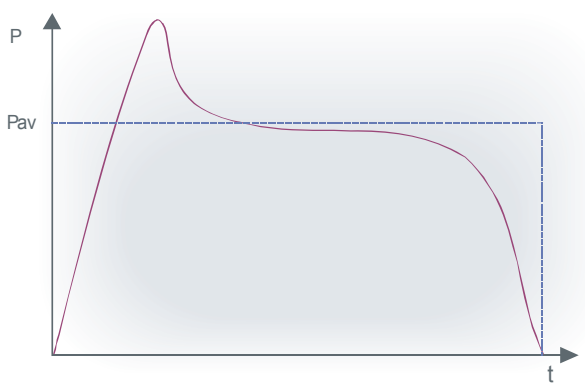

Figure 3: Thrust variations relative to time.

\subsection{Design criteria}

A criterion for the system's assessment is the requirement for low expenditure in fire put out. For the carrier however, the requirement has been for a minimum drag coefficient. Aerodynamic coefficients influencing the body's movement were the drag $\left(C_{D}\right)$; the lift force $\left(C_{L}\right)$; and the longitudinal momentum $\left(C_{m}\right)$ [3]. Since the carrier is an axially symmetrical body, the side force will be equal to the lift force as proved in Ashley and Landahl [5]. The axially symmetrical body overall resistance is determined by calculating its individual components separately. The drag coefficient for $\alpha=0$ attack angle, depends on the body shape, the Mach and the Reynolds number. The Mach number for a maximum velocity along the trajectory is $<0.52$. The coefficient will not practically be affected by this similarity criterion. Moreover, wave impedance is negligible for such velocities. The Reynolds number $\left(7.1 \times 10^{6}\right)$ is higher than the critical value of $6.5 \times 10^{6}$ and therefore a laminar boundary layer is being considered. The drag coefficient in the under sonic range is constant - 0.158. Comparisons drawn with literature [12] indicate that a $C_{D}=0.1975$ is a quality approximation.

\subsection{Design calculations}

Maximum velocity for both bodies will not go beyond $200 \mathrm{~m} / \mathrm{s}$. This is in compliance with Mach number, which equals to 0.54. It was assumed that during carrier fairing there will be no area where the local Mach number will go beyond 1. That is fairing will take place entirely in the under sonic range and there will be no wave impedance. Projectile studies have proven the relationship between the projectile shape and flight velocity, as illustrated in Fig. 4 [3].

Since the initial carrier velocity will be lower than $200 \mathrm{~m} / \mathrm{sec}$, it was assumed that a suitable body shape is illustrated on the left in Fig. 4 being suitable for the relatively high net volume it provides. The following correlations have been estimated between the geometric parameters:

$$
\begin{gathered}
l_{l}=1.324 d_{\text {mid }}(3), \quad l_{2}=d_{\text {mid }}(4), \quad r=2 d_{\text {mid }} \\
l=4.324 d_{\text {mid }}\left(l=l_{1}+3 d+d\right)
\end{gathered}
$$




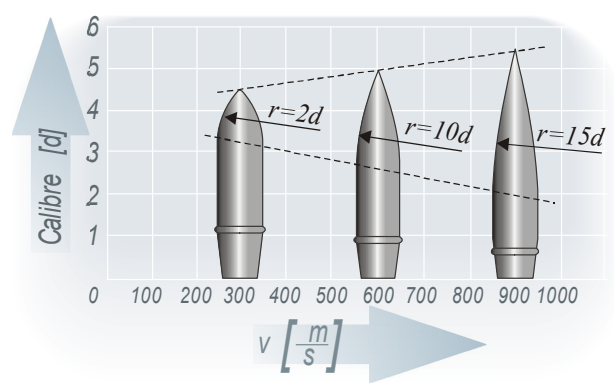

Figure 4: $\quad$ Relationship between projectile shape and flight velocity [3].

The $1_{1}$ value was determined by the Pythagorean Theorem geometry (Fig. 5).

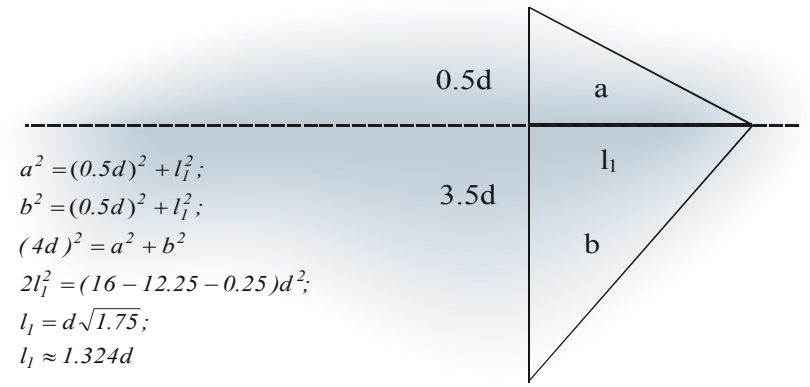

Figure 5: Determining the $l_{1}$ parameter.

Following diameter size modelling, the agent's net volume and carrier length results have been verified as shown in Table 1.

Table 1: Diameter size modelling.

\begin{tabular}{|c|c|c|}
\hline Diameter size d $[\mathrm{mm}]$ & Agent's net volume V $\left[\mathrm{dm}^{3}\right]$ & Carrier length $l[\mathrm{~mm}]$ \\
\hline 120 & 3 & 520 \\
\hline $\mathbf{1 4 0}$ & $\mathbf{5}$ & $\mathbf{6 0 5}$ \\
\hline 200 & 16 & 865 \\
\hline 300 & 54 & 130 \\
\hline $\mathbf{3 7 5}$ & $\mathbf{1 0 0}$ & $\mathbf{1 , 8 5 0}$ \\
\hline
\end{tabular}

\subsection{Comparison between the design variants}

\subsubsection{Comparing the projectile - missile solutions}

Figures 6 and 7 show the projectile and missile shape having the geometric parameters corresponding to the relations assumed above.

Above relations (3-6) have been assumed for the missile casing. The body length 1 is relative to motor's volume, thus the missile comprising a motor is of larger volume compared to the projectile. Larger volume provides for bigger 
external dimensions as length $l=1.933 \mathrm{~d}$ (Fig. 7), for the missile. Equation 3 illustrates that carrier calibre is large for artillery systems. Systems having a projectile calibre over $120 \mathrm{~mm}$ are rather heavy and not suitable for transportation in mountainous areas [6]. Missile-launching mechanisms of over $375 \mathrm{~mm}$ seem to be transported with greater mobility, while, the larger agent quantity carried makes the missile a more efficient carrier solution.

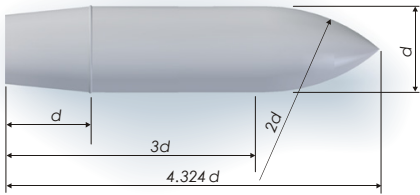

Figure 6: The projectile carrier shape.

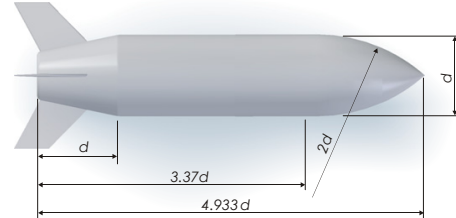

Figure 7: The missile carrier shape.

\subsection{Selection of the most suitable design}

The missile exhibits doubtless advantages over the projectile and it has been selected as the variant to be used for the final design.

\subsubsection{Determining missile's stability}

The exact elongation size should be determined in an aerodynamic experiment based on the static stability requirement [12]:

$$
\overline{x_{t}}-\overline{x_{f}}<-0.15 \text {, where: } \overline{x_{t}}=\mathrm{xt} / l \text { and } \overline{x_{f}}=\mathrm{xf} / l
$$

$\mathrm{x}_{t} \quad$ - distance between the centre of gravity and carrier front end;

$\mathrm{x}_{f}$ - distance between carrier focal point and carrier front end.

Table 2: $\quad$ The static stability requirement.

\begin{tabular}{|c|c|c|}
\hline $\begin{array}{l}\text { Distance between the centre of } \\
\text { gravity and carrier front end } \overline{x_{t}}\end{array}$ & $\begin{array}{c}\text { Distance between carrier focal } \\
\text { point and carrier front end }\end{array}$ & $\begin{array}{c}\text { Their difference } \\
\overline{x_{f}}-\overline{x_{f}}\end{array}$ \\
\hline 0.54 & 0.72 & -0.18 \\
\hline
\end{tabular}

For missile velocities up to $200 \mathrm{~m} / \mathrm{s}$ it is not necessary for stabilizers to have a $\chi$ arrow angle. Alternatively, it might prove necessary to use such an angle to provide for a longer stabilizer arm to ensure stability. A focal point is the point relative to which no change in the aerodynamic forces momentum is observed, when changing the attack angle. The missile's focal point and the mass centre have been determined and located parametrically and can be seen in Figure 8. 


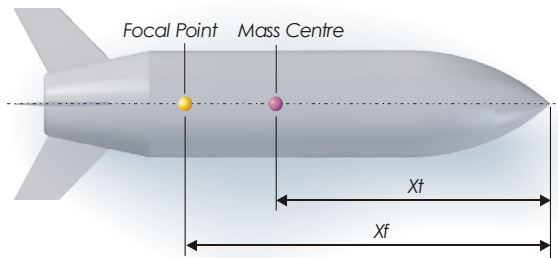

Figure 8: The missile's static stability.

\subsubsection{Determining missile's and agent's geometric parameters}

A missile carrier of a $100 \mathrm{dm}^{3}$ agent capacity has been selected amongst other variants. From Table 3 the calibre size $\mathrm{d}$ of the carrier relates to $375 \mathrm{~mm}$ for a $100 \mathrm{dm}^{3}$ missile. Other geometrical parameters, as the body length $l$ and individual component length were established and presented in Table 3.

Table 3: $\quad$ Body geometrical parameters.

\begin{tabular}{|c|c|c|c|c|}
\hline $\mathrm{V}\left[\mathrm{dm}^{3}\right]$ & $\mathrm{d}[\mathrm{mm}]$ & $l[\mathrm{~mm}]$ & $l_{l}[\mathrm{~mm}]$ & $l_{2}[\mathrm{~mm}]$ \\
\hline 100 & 375 & 1850 & 496.5 & 375 \\
\hline
\end{tabular}

A dimensionally detailed missile model is given in Fig. 9. The selected missile Drag coefficient has been estimated to be: $C_{D}=1.5 \mathrm{i}_{43}=0.237$.

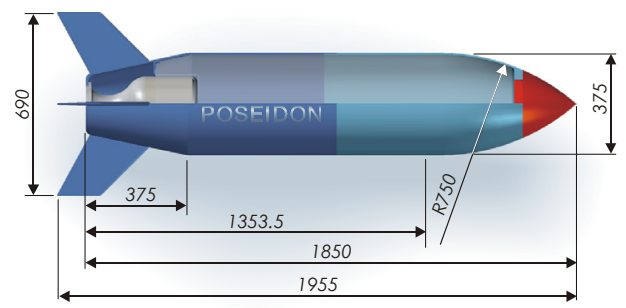

Figure 9: The missile's geometric parameters.

Carrier analysis has been done for essential geometry. The body maximum diameter allowed us to work out all other parameters as being of proportional value to the former. Body length has been found to be directly proportionate to the carrier calibre affecting flight stability, namely the long and short-term oscillations around its longitudinal axis [1]. An aerodynamic significant parameter is the relative "elongation", the ratio between body and calibre [3]. The larger the elongation, the lower the shape resistance coefficient. When the body's streamlined area increases the friction resistance increases respectively. 


\section{Movement's modelling}

\subsection{Boundary conditions and firing simulations}

Target coordinates, initial launching angle and firing direction can be determined when initial conditions are specified. When final conditions are specified, the solution lies in determining the insufficient initial conditions. The reverse problem [6] is solved directly through interpolation providing the initial velocity or thrust force data to achieve maximum flight distance.

\subsection{Movement along a specified basic trajectory}

\subsubsection{Velocity and acceleration and a function of time}

Simulations show small trajectory and velocity deviations in-between the bodies; the difference in flight time is also a negligible one. Notable variations however were observed in the acceleration as shown in Figure 10. The average acceleration for the whole second zero is low, $4.87 \mathrm{~m} / \mathrm{s}^{2}$. However, a zero second fraction is lengthier, several thousand $g$ times. Therefore, projectile's acceleration cannot go under several thousand g $(5,000 \mathrm{~g})$. The missile's acceleration is estimated through the engine's operation and varies widely, depending on the motor's operation, with the motor's acceleration for one second not exceeding $250 \mathrm{~m} / \mathrm{s}^{2}$ (25 times g). The motor's operation time could increase to $3 \mathrm{sec}$, leading to a maximum acceleration drop down to $10 \mathrm{~g}$.

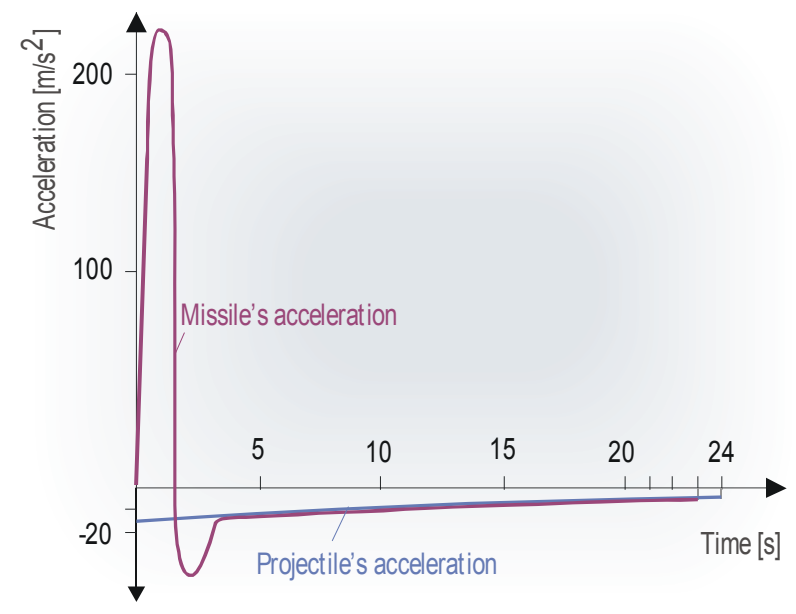

Figure 10: Acceleration as function of time.

\subsubsection{Distance as a function of launching angle}

The launching angle has an effect on the distance and the carrier's hitting accuracy, as shown in Figure 11. The distance dependence on the initial launching angle is a practical necessity for the system's application. Flight distance increases for angles close to 45 degree. For smaller angles, trajectories 


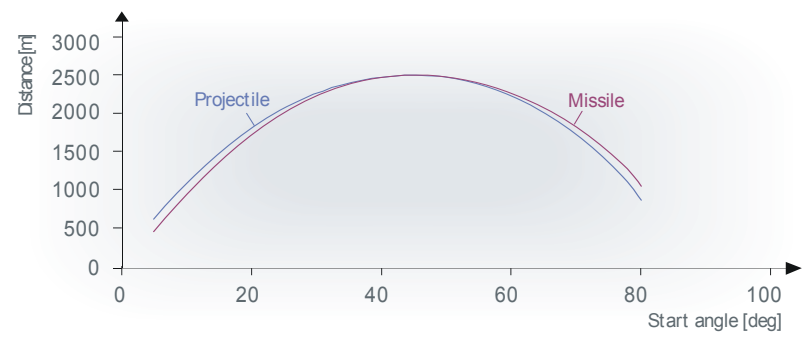

Figure 11: Distance as function of launching angle.

are flat curves and cannot be applied when high obstacles are in the way in mountainous areas. The longitudinal drift, in such trajectories is frequently significant. Therefore, trajectories resulting from launching acute angles are practically more convenient. For both carriers the maximum distance is achieved with an initial 45 degree launching. In both cases, firing can be performed with acute launching. Shorter distances can be reached with obstacles (heights) higher than the maximum trajectory height and acute launching.

\subsubsection{Trajectory for different agent masses}

Dependence between the required missile thrust and the extinguishing agent is linear as shown in Figure 12. Smaller calibre carriers will reach further as they feature lower drag characteristics. For agent mass $\leq 7 \mathrm{~kg}$, the system would be heavy and inconvenient to transport. Such limitations would occur for agents above $100 \mathrm{~kg}$ with missiles considered. The simulation has been performed with $100 \mathrm{~kg}$ missiles, expecting that with such agents extinguishing efficiencies will improve. To be able to achieve the maximum flight for larger calibres, it will be necessary to increase the projectile initial velocity or the motor thrust power on the missile. Simulations indicated that neither the resultant initial velocity for a $7 \mathrm{~kg}$ projectile, $164.6 \mathrm{~m} / \mathrm{s}$, nor the thrust power of a $100 \mathrm{~kg}$ agent missile $(14,3 \mathrm{~N})$, would present problems for variant development.

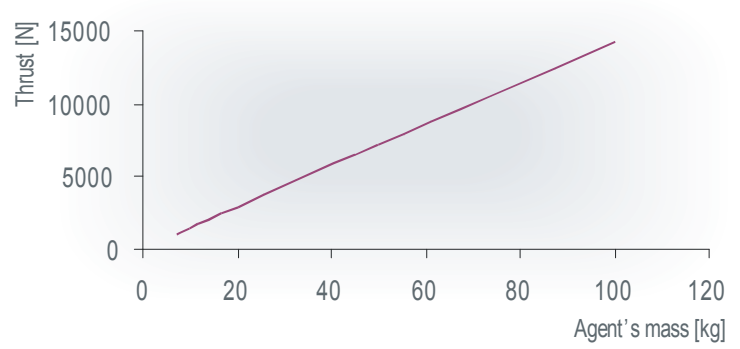

Figure 12: Dependence between thrust and extinguishing agent's mass. 


\subsection{Movement influenced by the environment}

\subsubsection{Longitudinal and cross wind effect}

Longitudinal wind affects the attained flight since it changes the carrier fairing speed, as shown in Figure 13 and 14. The flight distance increases with $20 \mathrm{~m}$ for tail wind velocities $+10 \mathrm{~m} / \mathrm{s}$. For higher wind velocities the flight distance increase came up to hundreds of meters. With the contrary wind present, the travel distance is reduced similarly.
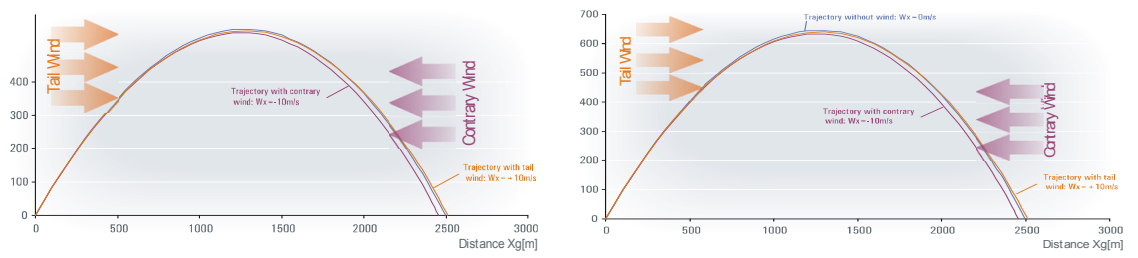
Figure 13: Dependence between Figure 14: Dependence between longitudinal wind and longitudinal wind and distance by the missile. distance by the projectile.

Figure 15 shows the dependence between cross wind and side drift. Cross wind would shift a projectile only throughout its entire flight in the same direction, acting on it by the side force. The shift on a missile will be against the wind while the motor is in operation. The side force acting on the pressure centre deflects its axis and thrust comprises a component force, the direction of which is against the wind [8]. The horizontal trajectory projection in-between the projectile and missile differ significantly with cross wind present.

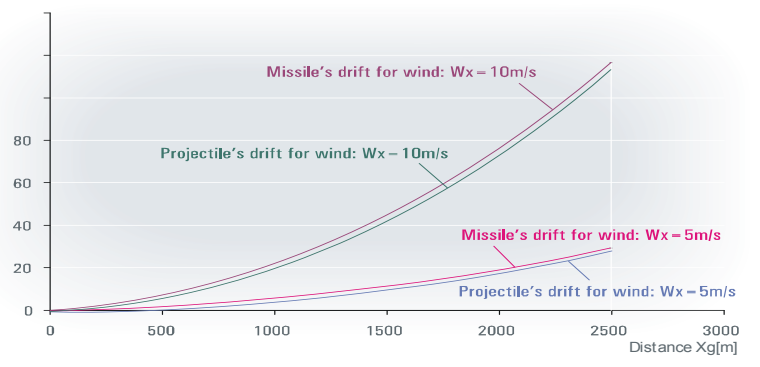

Figure 15: Dependence between cross wind and side drift for the missile and projectile.

\section{Conclusions}

The carrier shape has been selected on the requirements to ensure low drag during its movement, the dependence between shape and flight velocity and provision for the net volume required to carry the extinguishing agent. With the 
maximum carrier flight velocity being $200 \mathrm{~m} / \mathrm{s}$, the most appropriate shape came through the adoption of a projectile for flight velocities under $300 \mathrm{~m} / \mathrm{s}$.

When the projectile is fired, acceleration exceeds 5,000 times gravity necessitating that rigid materials, often steel are used, in which body wall thickness is impressive. Missile accelerations usually do not exceed $25 \mathrm{~g}$. When a projectile is used the target hitting accuracy is high. Nevertheless, this is not a significant advantage when extinguishing mountainous fires, where one would expect a surface area target and not a particular point. A projectile disadvantage is the limited extinguishing agent quantity to be carried.

Virtually, a projectile carrier cannot be used when agent quantities of above $5 l$ are involved and this lowers the efficiency of a projectile, hence the increased number of carriers required to put out a fire. The missile exhibits doubtless advantages and it has been selected, as the variant to be used for further system development. It provides for carrying agent quantities of far over the pre-set value of $5 \mathrm{l}$. Consequently, a missile carrier of a $100 \mathrm{l}$ capacity could provide for improvement of the overall system efficiency.

\section{References}

[1] Xanthopoulos, G., Forest fires: Past present \& future. Epikentra, 6, 62-71, 1998.

[2] Kailidis, D., Xanthopoulos G., The forest fire problem. Aristotelian university of Thessaloniki, Greece, Department of forestry and natural environment, Forest protection laboratory report: (ERIC No. 3: 10-12), 1998.

[3] Conference on Fire fighting equipment \& materials, Materials \& design against fire. 27-28 October 2002, Institution of mechanical Engineers, Mechanical Engineering publications, London: 23-28, 2002.

[4] McCormick, B., Aerodynamics, Aeronautics, and Flight Mechanics. (2nd Ed.). Pennsylvania State University: John Wiley and Sons, Inc, 1995.

[5] Ashley, H., Landahl, M., Aerodynamics of Wings and Bodies. Dover: Dover Publications, 1995.

[6] Anderson, J. D., Introduction to Flight. London: McGraw-Hill Inc, 1999.

[7] Glor, M., Hazards in fire handling. Cambridge: Harvard university press, 1999.

[8] Houghton, J., The Physics of Atmospheres. Cambridge: Cambridge University Press, 2001.

[9] Cote, A. E., Linville, J. L., Fire Protection Handbook, (17th ed.), London: National fire protection Association: 49-56, 1991.

[10] Dean, A. E. Tower, K., Fire protection guide, (10th Ed), London: National fire protection Association: 198-205, 1994.

[11] Anderson, J., John, D., Fundamentals of Aerodynamics. London: McGrawHill, Inc, 2001.

[12] Jensen, G. E.; Netzer, D. W., Tactical missile propulsion. American institute of Aeronautics and Astronautics. Massachusetts: AIAA, Inc, 1996. 